\title{
STUDY OF RHEOLOGICAL PROPERTIES OF BITUMINOUS BINDERS IN MIDDLE AND HIGH TEMPERATURES
}

\author{
Eva REMIŠOVÁ ${ }^{1,{ }^{*}}$, Viera ZATKALIKOVÁ ${ }^{2}$, František SCHLOSSER $^{3}$
}

1 Department of Highway Engineering, Faculty of Civil Engineering, University of Žilina, Univerzitná 8215/1, 01026 Žilina, Slovakia.

2 Department of Materials Engineering, Faculty of Mechanical Engineering, University of Žilina, Univerzitná 8215/1, 01026 Žilina, Slovakia.

${ }^{3}$ Department of Construction Management, Faculty of Civil Engineering, University of Žilina, Univerzitná 8215/1, 01026 Žilina, Slovakia.

corresponding author: eva.remisova@fstav.uniza.sk.

\section{Abstract}

The bitumen binders in road pavements are exposed traffic loading effect at different climatic conditions. A resistance to these stresses depends on bitumen properties as well. The paper presents rheological properties $\left(G^{*}, \delta, \eta^{*}\right)$ determined and compared for four bituminous binders (unmodified and polymer modified bitumen) at temperature $46-60(80)^{0} \mathrm{C}$ and dynamic viscosity at temperature $130-190^{\circ} \mathrm{C}$ (Brookfield viscometer). On the basis of viscosity results it is possible to set optimal production and compaction temperatures. Elastic and viscous behavior of binder in the middle temperature is determined in rheometers. The higher value of complex modulus, the stiffer bitumen binder is able to resist deformation. The greater content of elastic components (e.g. polymer in bitumen) varies mainly elastic-viscous properties of primary bitumen.
\end{abstract}

\author{
Keywords: \\ Bitumen; \\ Complex shear modulus; \\ Viscosity; \\ Rheology; \\ Temperature.
}

\section{Introduction}

Bitumens as well as asphalts are exposed to various chemical and mechanical stresses in road pavement, as stress, tension, bending, impact and several effects together. Therefore, it is important to know how the bitumen is resistible to that stress (what a rate, what conditions). Bitumen is a colloidal system including two different groups of colloidal dispersed parts. The asphaltenes and aromatic resins (stabilizing dispersion of asphaltenes) create the first group. The second group consists of maltenes (oil part of bitumen) [1]. The bitumen properties are derived from their colloidal structure and chemical composition. The basic tests as penetration and softening point are dedicated to evaluate and classify unmodified bitumens. With the increased use of bitumen modifications such as polymers and mastics, the fundamental approach is required regarding the performance-based tests [2]. A deformation and flow behavior of materials are determined in rotational and oscillatory measuring systems. The rheometers allow measuring of qualitative and quantitative relations between strain and stress by means oscillatory-type testing.

Bitumen binders in the medium to high temperature range behave partly as viscoelastic materials. A viscoelastic material has both elastic and viscous components of response: effecting of external force influence there is partly a permanent deformation (viscous part) and partly a reversible deformation (elastic part). One of the main aims of rheology is determining the relation between stains and stresses. The rheological properties of bitumen binders that have influence on material deformation and flow are generally expressed in term of the complex viscosity $\eta^{*}$, the complex modulus $G^{*}$ and the phase angle $\delta$. The viscosity expresses a resistance constant of material (a liquid, a bitumen) to deformation under the influence of shear stress. This internal friction is coming up when there is a movement of materials layers with one another (shear stress of unit element); see Fig.1. Viscosity is a value of bitumen consistency at specific shear stress and specific temperature and it characterizes structural and thixotropic properties of bitumen. At constant shear stress and at different temperature the viscous curves that characterized bitumen temperature sensitivity are determined. 
From relation between viscosity and shear stress at a specific temperature the structural viscosity characteristic is obtained. The shear modulus is a material specific value that expresses the ratio of the shear stress and corresponding deformation [3].

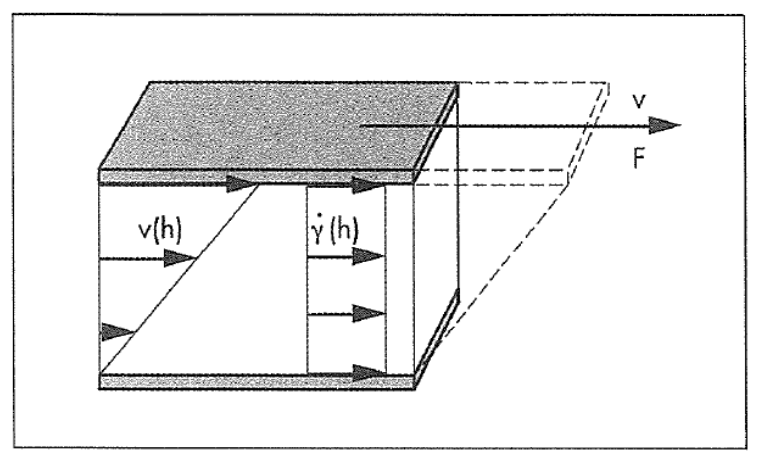

Fig. 1: Two plate model for shear tests to illustrate the velocity distribution of a flow in the shear gap [3].

The bitumen technological processing is closely associated with rheological characteristics. Shearing occurs during bitumen pumping, spraying, mixing, compacting etc.

Understanding the flow and deformation characteristics of bitumens is important from the pavement performance point of view. Using the bitumen that deforms and flows at standard service temperatures decreases a resistance of the asphalts in pavement to permanent deformation and rutting. On the other side too stiff bitumen may be exposed to fatigue and cracking [2]. The use of quality binder is one of the requirements for the satisfactory pavement performance.

\section{Proposed methodology}

\subsection{Laboratory tests description}

For the purpose of evaluate of bitumen behavior at medium and high temperatures, the paving grade binders and modified binders were tested in an oscillatory rheometer. In rheometer a thin bitumen sample is sandwiched between two parallel plates (Parallel-plate measuring system PP MS). The bottom plate is stationary. The oscillatory motion of upper plate causes a shearing of sample. A sinusoidal oscillatory shear stress is used to a sample and the resulting shear strain is measured. The applied sinusoidal varying stress $\tau$ is expressed:

$\tau(t)=\tau_{0} \cdot \sin \omega t$

and the resulting strain as:

$\gamma(t)=\gamma_{0} \cdot \sin (\omega t+\delta)$

where $\tau_{0}$ is the stress amplitude $(\mathrm{Pa}), \omega$ angular frequency $\left(\mathrm{rad}_{\mathrm{s}} \mathrm{s}^{-1}\right), t$ time $(\mathrm{s}), \gamma_{0}$ the strain amplitude and $\delta$ the phase angle of the measured material between the preset and the resulting curve $\left({ }^{\circ}\right)$.

The complex shear modulus $G^{*}(\mathrm{~Pa})$ as the resistance to deformation is defined as a ratio of the values of the sinusoidal functions of $\tau(t)$ and $\chi(t)[3,4]$ :

$G^{*}=\frac{\tau(t)}{\gamma(t)}$

Parameters, resulting from harmonic-periodic processes, always should be written in the complex form [3]:

$G^{*}=G^{\prime}+i \cdot G^{\prime \prime}$

The real part, the storage modulus $G^{\prime}$, represents elastic behavior of material which characterizes the energy stored during a shear process. The imaginary part, the loss modulus $G^{\prime \prime}$, 
represents the viscous behavior of material which characterizes the energy dissipated during a shear cycle. The resultant of two components $G^{\prime}$ and $G$ " characterizes the complete viscoelastic behavior which is composed of both elastic and viscous parts.

The complex viscosity $\eta^{*}$ (Pa.s) determined by oscillatory test is defined as the ratio:

$\eta=\frac{\tau(t)}{\gamma(t)}$

The viscosity, as one of fundamental characteristics of liquids, assesses the material behavior at different temperatures. The coaxial cylinder measuring system (CC MS) is used to obtain a continuous viscosity-temperature curve. The torque applied to a spindle rotating and immersed in a sample, measures the resistance of a spindle to rotation and provides a measure of the dynamic viscosity $\eta$ of the sample. The applied shear stress $\tau$ is calculated according:

$\tau=\frac{M}{2 \pi R_{b}^{2} L}$

and shear rate $\gamma$ related to the spindle surface as:

$\dot{\gamma}=\frac{2 \omega R_{c}^{2} R_{b}^{2}}{x^{2}\left(R_{c}^{2}-R_{b}^{2}\right)}$

where $\omega$ is angular velocity of spindle $\left(\mathrm{rad}_{\mathrm{s}} \mathrm{s}^{-1}\right), R_{c}$ is container radius $(\mathrm{cm}), R_{b}$ is spindle radius $(\mathrm{cm})$, $x$ is distance between rotation axis and any layer of the sample (cm), $M$ is torque (dyn-cm) and $L$ is active spindle length $(\mathrm{cm})$. The calculated value of ratio between the applied shear stress and the shear rate represents the dynamic viscosity.

\subsection{Materials}

The tested materials included binders used in asphalt mixtures built-in pavement constructions, two unmodified bitumens $(50 / 70,70 / 100)$ and three various polymer modified bitumen (Kraton, Sealoflex and Apollobit). Properties of used bitumen binders are in Table 1.

Table 1: The empirical properties of tested bitumens.

\begin{tabular}{|l|c|c|c|c|c|}
\hline Bitumen properties & Kraton & Sealoflex & CA 50/70 & CA 70/100 & Apollobit \\
\hline Penetration $(0.1 \mathrm{~mm})$ & 82 & 71.5 & 58 & 78 & $50-100$ \\
\hline Softening point $\left({ }^{\circ} \mathrm{C}\right)$ & 82,8 & 89 & 48.2 & 48 & min. 70 \\
\hline Penetration index $(-)$ & 6,2 & 6.5 & 1.3 & $-0,6$ & - \\
\hline
\end{tabular}

\subsection{Experimental conditions}

The rheology tests were performed according to EU specification EN 14770 [5] using the oscillatory rheometer (Physica MCR301) with temperature control attachment CTD 450 (Fig. 2), under the following test conditions:

- test sample of bitumen placing between parallel plates of rheometer (PP25 system) with $25 \mathrm{~mm}$ in diameter and a small gap of $1 \mathrm{~mm}$,

- the test temperatures $40,46,50,54,60$ and $80^{\circ} \mathrm{C}$,

- amplitude of deformation $5 \%$,

- angular frequencies in the range of $30-200 \mathrm{rad}^{-1}$ at temperatures $46,50,54$ and $60^{\circ} \mathrm{C}$, and in range $30-600$ rad. s $^{-1}$ at temperatures 60 and $80^{\circ} \mathrm{C}$.

The dynamic viscosity $\eta$ of binder at high temperatures about $130-180^{\circ} \mathrm{C}$ was measured according to EU specification EN 13302 [6] using a rotating spindle apparatus Brookfield viscometer model DV-II+Pro with cylindrical spindle and thermoset system (Fig. 2). Different binders were tested at a constant temperature with spindle SC4-27 while varying the spindle speed. 


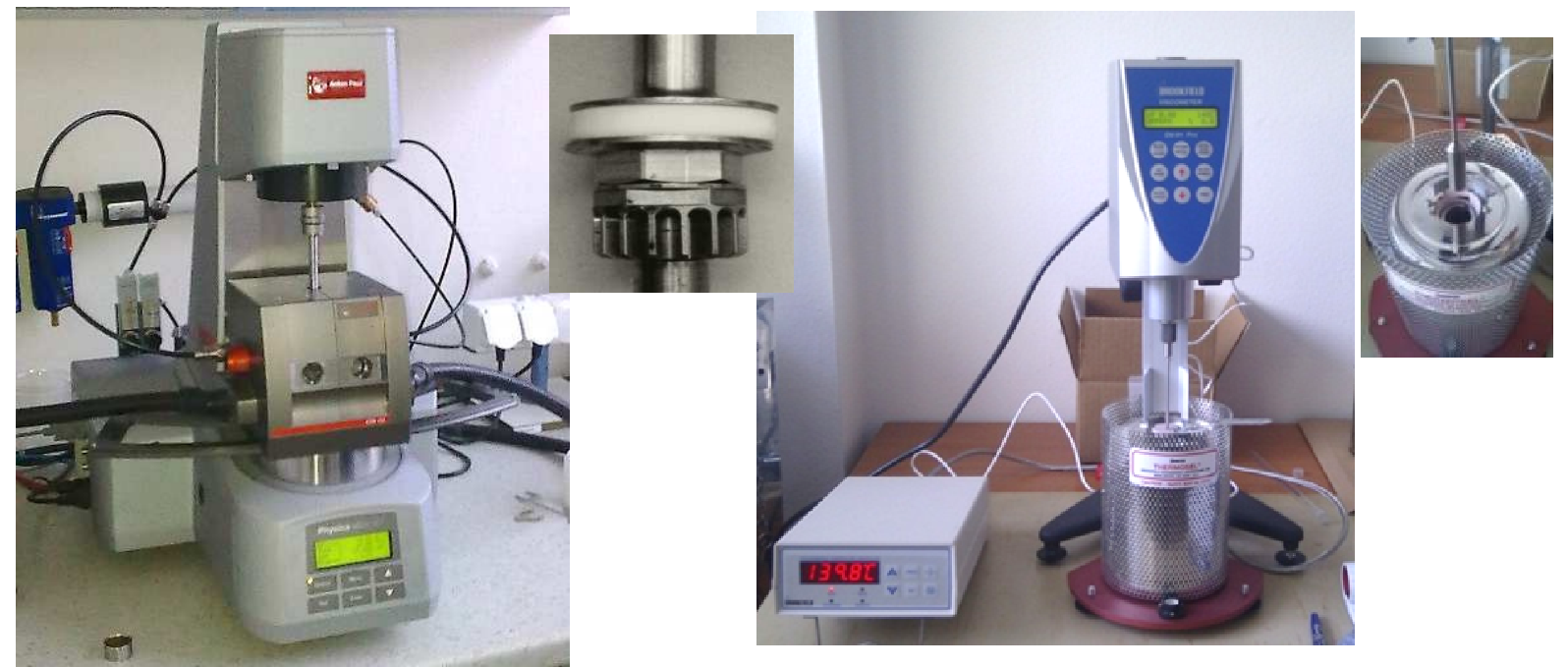

Fig. 2: PP MS system, Physica Rheometer MCR301 (left) and CC MS system, Brookfield Viscometer DV-II+Pro, spindle SC4-27 (right).

\section{Results and analysis}

The rheological characteristics, studied in dynamic shear rheometer and viscometer, are showed in next Fig. 3, 4 and 5. A comparison of rheological parameters of all tested bituminous binders at the temperature of $46^{\circ} \mathrm{C}$ shows that plots of moduli $G^{\prime}, G^{\prime \prime}$ and complex viscosity of bitumen Apollobit are upper than other binders (Fig. 3). Rheological parameters of other two modified binders Sealoflex and Kraton are similar to parameters of unmodified 50/70. The lowest values of complex viscosity and both moduli are achieved by paving grade bitumen 70/100. At temperature $60^{\circ} \mathrm{C}$, the values of storage modulus and complex viscosity of unmodified binders are markedly lower than the values of modified ones (except for complex viscosity at higher angular frequencies $\omega 500-$ $600 \mathrm{~s}^{-1}$ ). Values of rheological parameters of modified binder Apollobit remain the highest as well. At both temperatures, the modified binders show sharper differences between complex viscosity at angular frequency $30 \mathrm{~s}^{-1}$ and frequency $200 \mathrm{~s}^{-1}$ than unmodified ones.

Modified binders were tested at $80^{\circ} \mathrm{C}$ as well (Fig. 3). The values of parameters $G^{\prime}, G^{\prime \prime}$ and $\eta^{*}$ decreased at temperature $80^{\circ} \mathrm{C}$. At this temperature, the dependency of storage modulus $G^{\prime}$ on frequency in interval 400 to $600 \mathrm{~s}^{-1}$ loses linearity, the values of modulus $G^{\prime}$ decrease and it marks the loss of binder elasticity and degradation.

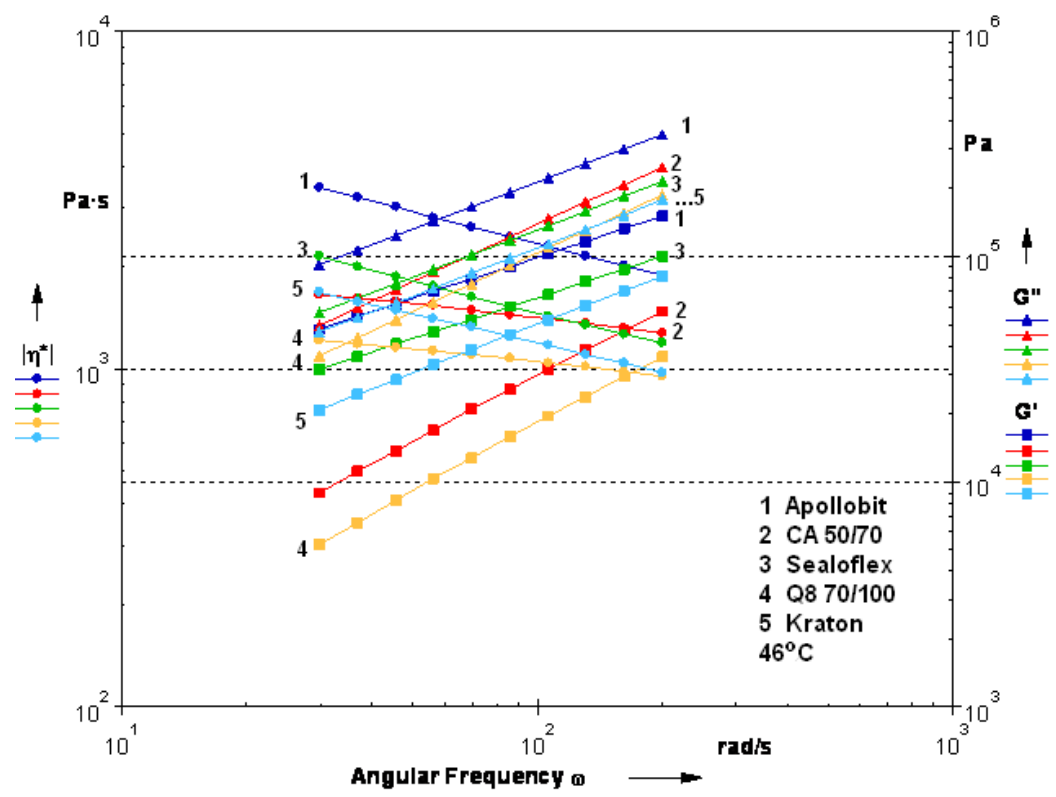



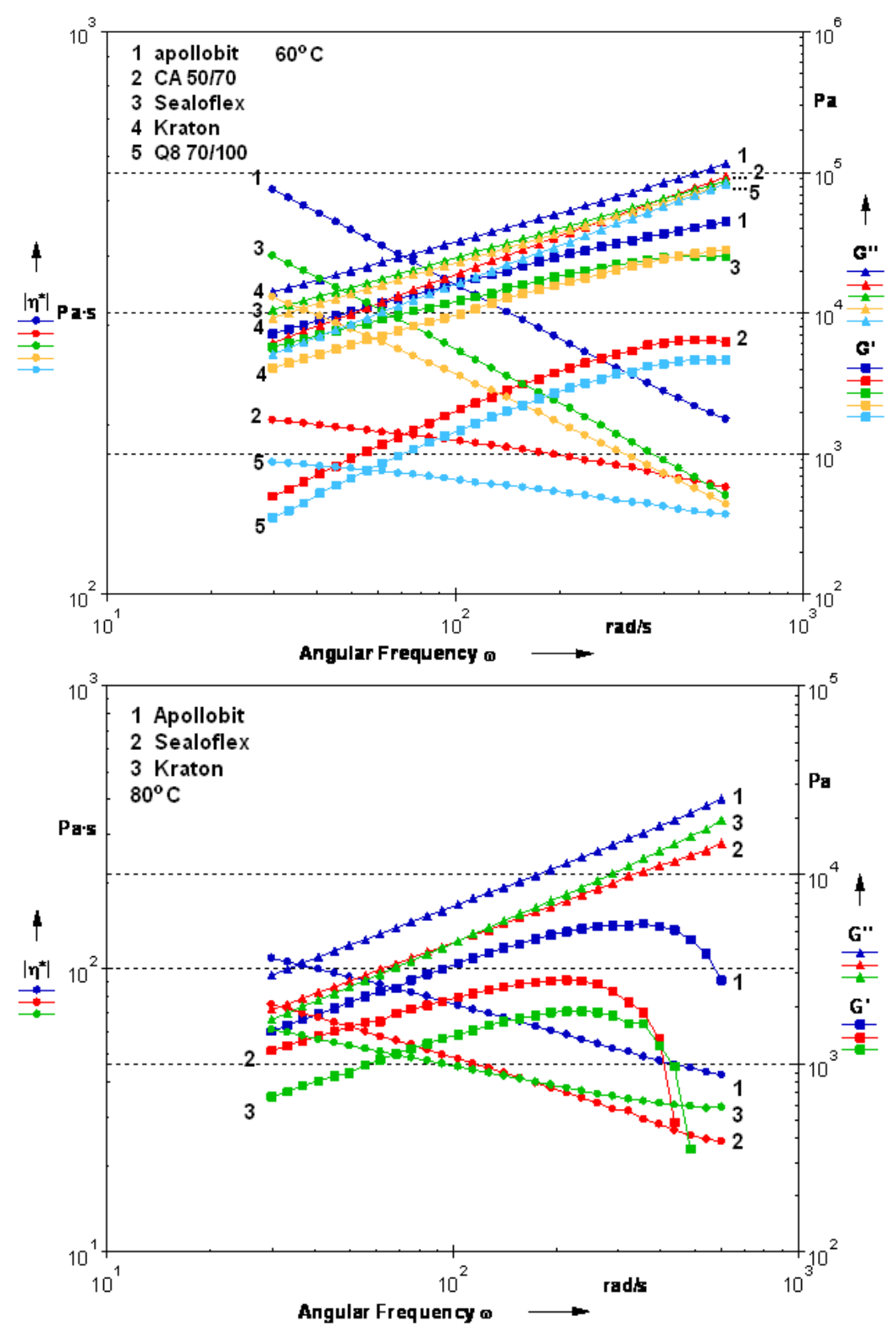

Fig. 3: Comparison of rheological parameters of tested binders at temperatures 46,60 and $80^{\circ} \mathrm{C}$ [7].

The procedure in DSR involves to evaluate a value of complex shear modulus $G^{*}$ and phase angle $\delta$ (the lag within the applied shear stress and the resulting shear strain) of bitumens at various test frequencies and temperatures. The measurements show the phase angle is responsive to type and chemical structure of bitumen as [10] present. In case unmodified bitumens (50/70, 70/100) phase angle is larger (Fig. 4) and characterizes a bitumen as more viscous material. The modification of bitumen improves elastic response (reduced phase angle). The results of complex modulus $G^{*}$ show an increase with load frequency and a decrease with temperature. The modified binder Appolobit reached the highest values of complex modulus at all tested temperatures. Compared to unmodified binders, the increase in $G^{*}$ for modified bitumens is given by storage modulus $G^{\prime}$ that is associated with the elastic part of material behavior (Fig. 3). This signifies the modified bitumens are more resilient and a bit stiffer than unmodified binders. At temperature $80^{\circ} \mathrm{C}$, the increasing loading frequency the bitumen more viscous behavior. 

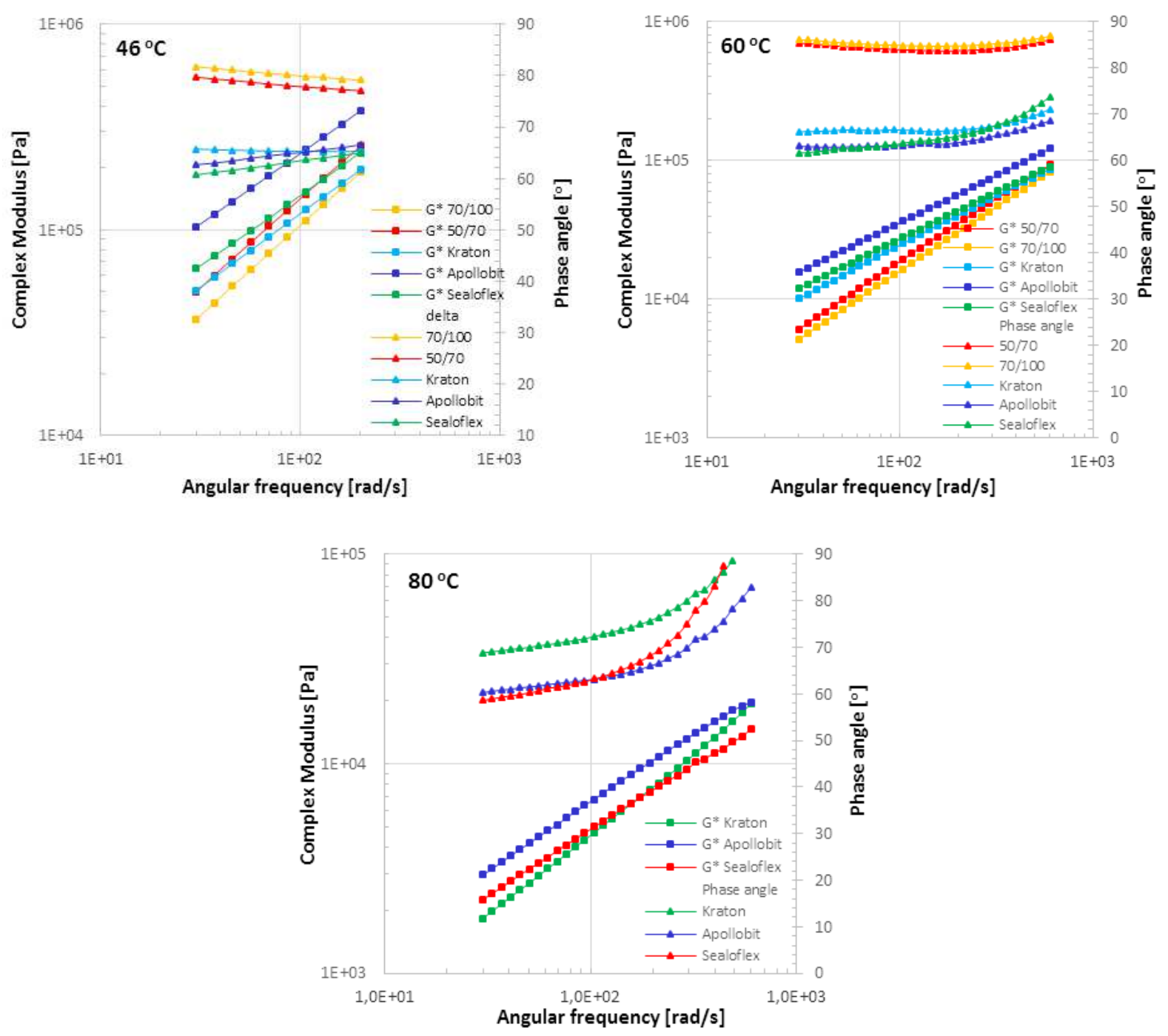

Fig. 4: The complex shear modulus and its phase angle of tested binders at temperatures 46,60 and $80^{\circ} \mathrm{C}$.

The dynamic viscosities of binders are determined using a rotating spindle apparatus. The applied torque making rotation of a spindle in a container containing the test sample measures the related resistance of the spindle to movement and provides a measure of the dynamic viscosity of the sample.

The results of dynamic viscosity of selected binders (empirical characteristics in Table 1) at temperature in range 130 to $190^{\circ} \mathrm{C}$ are shown in Fig. 5. For current viscometer torque constant (T) and spindle constants (spindle multiplier constant SMC and shear rate constant SRC) was determined shear rate (in s ${ }^{-1}$ ) and dynamic viscosity (in $\mathrm{mPa} . \mathrm{s}$ ) at set temperature and velocity of spindle. The results show that the viscosity for all binders decreases as the increase of temperature. A sharp decline in viscosity is indicated as the change of temperature $130^{\circ} \mathrm{C}$ and $140^{\circ} \mathrm{C}$. Modified binders have a higher viscosity than unmodified bitumens $(50 / 70,70 / 100)$. At high temperatures, the bitumens behave as newton fluids. 

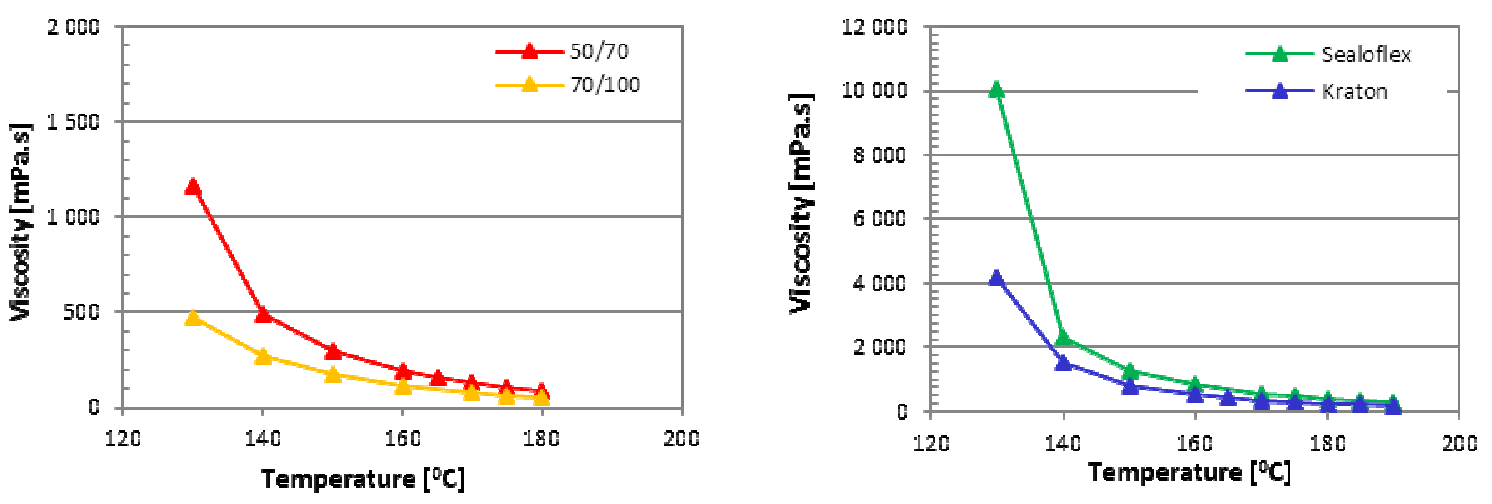

Fig. 5: Dynamic viscosity unmodified and modified bitumens.

Fig. 6 shows the temperature-viscosity plot unite the dynamic viscosities (Brookfield viscometer) and complex viscosities (oscillatory test, the dynamic shear rheometer). The viscosities at medium temperatures characterize binder at temperature typically occurs in pavement surface during summer. The viscosity measurements at high temperatures enable to assess temperatures proper workability of binder (pumping, mixing and compaction). The representative dynamic viscosity values for bitumens are from 0.2 to 2 Pa.s at these temperatures. The bitumen binders at in-service temperatures of pavement achieve the dynamic viscosity value about 200 Pa.s [8]. The low viscosity of bitumen binders requires material heating to high production temperatures exceeding $150{ }^{\circ} \mathrm{C}$. Similar effect, considering production-related emissions of greenhouse gases and their negative effect on the environment, reaches by use additives (Fischer-Tropsch wax, montan wax, fatty acid amides, chemical surface active additives), water (foamed bitumen), hydrophilic material such as zeolite [9].

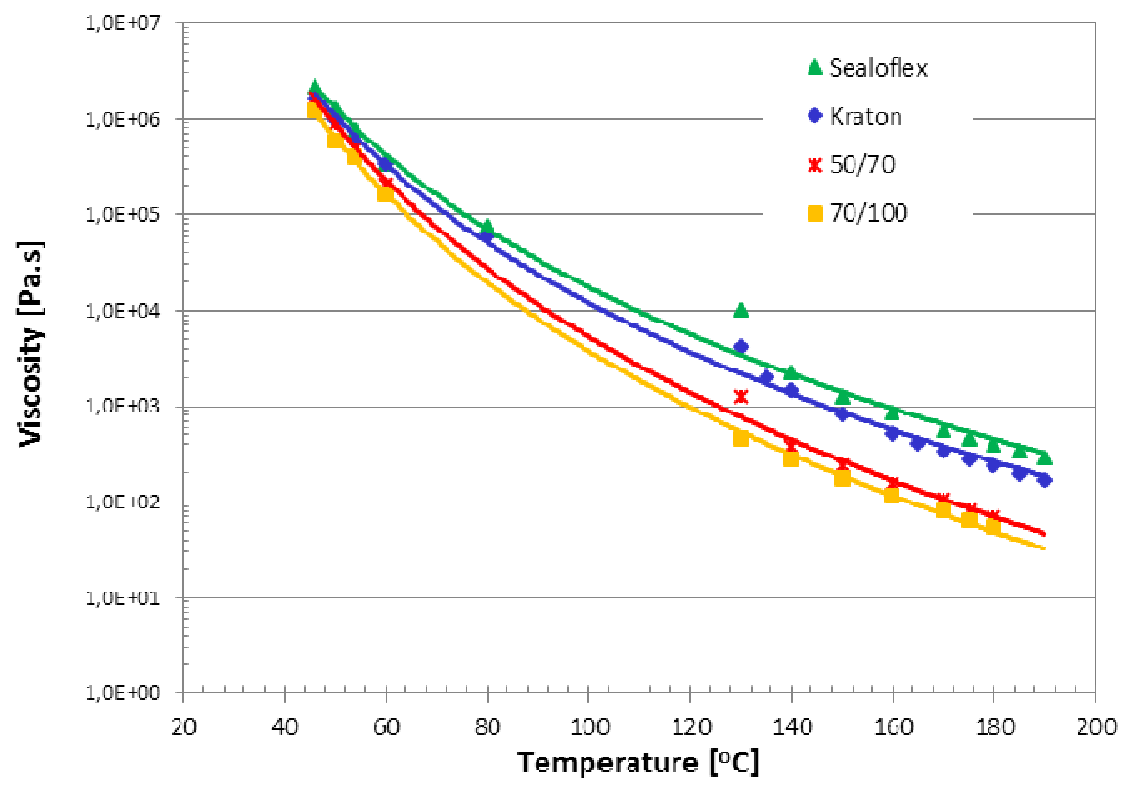

Fig. 6: Viscosity-temperature relationship of tested binders in temperature range 46 and $190^{\circ} \mathrm{C}$.

\section{Conclusions}

The rheological measurements allow the study of behavior of bitumens (elastic and viscous) at various temperatures and to compare different binders at same conditions.

Rheological parameters $G^{\prime}, G^{\prime \prime}, \eta^{*}$ of tested bitumen binders are strongly affected by temperature. At given test frequencies and temperatures binder Apollobit (PMB) achieved higher values of rheology parameters then other tested binders. The lowest values of rheological parameters were marked with unmodified binder 70/100. 
The modified bitumens showed expressive decrease of complex viscosity depending on development of angular frequency as compared to unmodified.

The binders modified by polymer mostly show a higher value of complex modulus and a lower value of phase angle. That shows the binders have more elasticity and stiffness than unmodified binders.

On the base of test results and bitumen behavior at various temperatures it is able to suppose the asphalts built-in pavement will be resist to permanent deformation during hot weather.

The relation within the elastic and viscous behavior of binders is used to predict their performance in paving applications.

\section{References}

[1] READ, J. - WHITEOAK, D.: The Shell Bitumen Handbook. 4th edition. Thomas Telford, London.

[2] NUR IZZI Md. YUSOFF et al.: Modelling the linear viscoelastic theological properties of bituminous binders. In: Construction and Building Materials. Vol. 25 (2011), ISSN 2171-2189.

[3] MEZGER, T. G.: The Rheology Handbook. 2nd edition. Vincentz Network 2006, ISBN 3-87870174-8.

[4] KAHOVEC, J.: Rheology of Polymer Systems. Wiley-VCH, Praha, 2000, p. 182.

[5] STN EN 14770:2012 Asfalty a asfaltové spojivá. Stanovenie komplexného modulu v strihu a fázového uhla. Dynamický strihový reometer (DSR).

[6] STN EN 13302:2010 Asfalty a asfaltové spojivá. Stanovenie dynamickej viskozity asfaltových spojív rotačným vretenovým viskozimetrom.

[7] ZATKALÍKOVÁ, V. - SCHLOSSER, F. - REMIŠOVÁ, E.: Comparison of theological parameters of selected polymer modified bituminous binders. In: Acta Technica Corviniensis - Bulletin of Engineering. Tom 7, Fasc. 3, 2014. ISSN 2067-3809.

[8] WEST, R.C. et al.: Mixing and Compaction Temperatures of Asphalt Binders in Hot-Mix Asphalt. Report 648. National Academy of Sciences, 2010.

[9] CHOMICZ-KOWALSKA, A.: Statistical methods for evaluating associations between selected foamed bitumen parameters. In: Proceedings of the 6th International Conference on Bituminous Mixtures and Pavements - ICONFBMP 2015. CRC Press/Balkema, p. 3 - 12.

[10] AIREY, G. D.: Styrene butadiene styrene polymer modification of road bitumens. In: Journal of Materials Science. Vol. 39 (2004), p. 951-959. 